\title{
Unit of Infective Dose
}

National Cancer Institute

\section{Source}

National Cancer Institute. Unit of Infective Dose. NCI Thesaurus. Code C70475.

A potency unit for measuring infectious activity of biologic material defined as a minimal dose at which an infectious agent contained in this material can reproduce in the host or the system in which it has been inoculated and to produce specific and measurable effect(s). 